\title{
On the $\sin \phi_{R}$ single longitudinal spin asymmetry in dihadron production in SIDIS
}

\author{
Wei Yang ${ }^{* \dagger}$ \\ School of Physics, Southeast University, Nanjing 211189, China \\ E-mail: weiyang@seu.edu.cn
}

\section{Zhun Lu}

School of Physics, Southeast University, Nanjing 211189, China

E-mail: zhunlu@seu.edu.cn

\begin{abstract}
We study the single longitudinal-spin asymmetry of dihadron production in semi-inclusive deep inelastic scattering process in which the transverse momentum of the final-state hadron pairs is integrated out. In Particular, we investigate origins of the $\sin \phi_{R}$ azimuthal asymmetry for which we take into account the coupling of the twist-3 distributions $h_{L}$ and the dihadron framgentation function (DiFF) $H_{1, \text { ot }}^{\varangle}$ as well as the coupling of the helicity distribution $g_{1}$ and the twist-3 DiFF $\widetilde{G}^{\varangle}$. To this end The unknown twist-3 dihadron fragmentation function $\widetilde{G}^{\varangle}$ is calculated in a spectator model which is successful in describing the dihadron production in unpolarized process. We estimate the $\sin \phi_{R}$ asymmetry of dihadron production in SIDIS at the kinematics of COMPASS and compare it with the preliminary COMPASS data. Although the asymmetry is dominated by the $h_{L} H_{1}^{\varangle}$ term, we find that the contribution from the $g_{1} \widetilde{G}^{\varangle}$ term should also be taken into account in certain kinematical region.
\end{abstract}

XXVII International Workshop on Deep-Inelastic Scattering and Related Subjects - DIS2019

8-12 April, 2019

Torino, Italy

\footnotetext{
* Speaker.

${ }^{\dagger}$ This work is partially supported by the NSFC (China) grant 11575043, by the Fundamental Research Funds for the Central Universities of China.
} 


\section{Introduction}

The azimuthal asymmetries in semi-inclusive deep inelastic scattering (SIDIS) process have been recognized as useful tools for these quests. The full description of SIDIS includes a set of parton distribution functions (PDFs) and fragmentation functions (FFs). [1, 2]. The unpolarized DiFFs were introduced in Ref. [3], and their evolution equations have been investigated in Refs. [4, $5,6]$. Particularly, the chiral-odd $\operatorname{DiFF} H_{1}^{\varangle}[7,8,9]$ plays an important role in accessing transversity distribution, as it couples with $h_{1}$ at the leading-twist level in the collinear factorization. In this work, we study the $\sin \phi_{R}$ asymmetry by adopting the spectator model results for the distribution functions and fragmentation functions. We not only take into account the coupling $h_{L} H_{1}^{\varangle}$, but also investigate the role of the T-odd DiFF $\tilde{G}^{\varangle}$, which encodes the quark-gluon-quark correlation and has not been considered in previous studies.

\section{Formalism of the $\sin \phi_{R}$ asymmetry of dihadron production in SIDIS}

As displayed in Fig.1, the process under study is the dihadron production in SIDIS off a longitudinally polarized proton target:

$$
\mu(\ell)+p^{\rightarrow}(P) \longrightarrow \mu\left(\ell^{\prime}\right)+h^{+}\left(P_{1}\right)+h^{-}\left(P_{2}\right)+X,
$$

where the four-momenta of the incoming and the outgoing leptons are denoted by $\ell$ and $\ell^{\prime}, P$ is the momentum of the target with mass $M$. In this process, the active quark with momentum $p$ is struck by the virtual photon with momentum $q=\ell-\ell^{\prime}$. The final-state quark with momentum $k=p+q$ then fragments into two final-state hadrons, $h^{+}$and $h^{-}$, plus unobserved state $X$. The momenta of the pair are denoted by $P_{1}, P_{2}$.

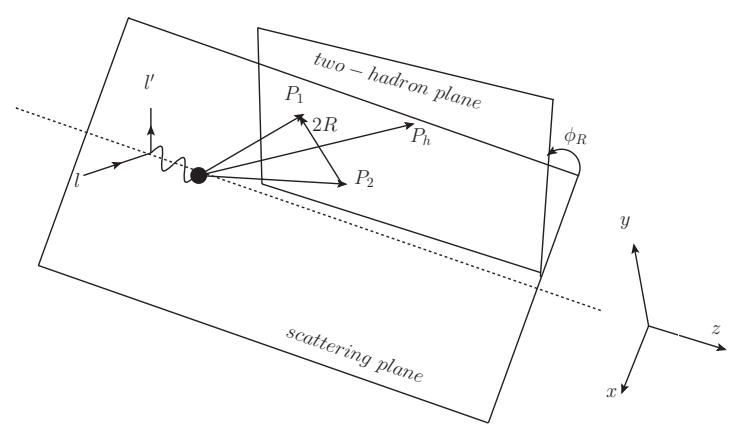

Figure 1: Angle definitions involved in the measurement of the single longitudinal-spin asymmetry in deepinelastic production of two hadrons in the current region.

The twist-3 DiFF $\widetilde{G}^{\varangle}$ arises from the multiparton correlation during the quark fragmentation, described by the quark-gluon-quark correlator $[13,15]$ :

$$
\begin{aligned}
\widetilde{\Delta}_{A}^{\alpha}\left(z, k_{T}, R\right)= & \frac{1}{2 z} \sum_{X} \int \frac{d \xi^{+} d^{2} \xi_{T}}{(2 \pi)^{3}} e^{i k \cdot \xi}\langle 0| \int_{ \pm \infty^{+}}^{\xi^{+}} d \eta^{+} \mathscr{U}_{\left(\infty^{+}, \xi^{+}\right)}^{\xi_{T}} \\
& \times\left. g F_{\perp}^{-\alpha} \mathscr{U}_{\left(\eta^{+}, \xi^{+}\right)}^{\xi_{T}} \psi(\xi)\left|P_{h}, R ; X\right\rangle\left\langle P_{h}, R ; X\left|\bar{\psi}(0) \mathscr{U}_{\left(0^{+}, \infty^{+}\right)}^{0_{T}} \mathscr{U}_{\left(0_{T}, \xi_{T}\right)}^{\infty^{+}}\right| 0\right\rangle\right|_{\eta^{+}=\xi^{+}=0, \eta_{T}=\xi_{T}} .
\end{aligned}
$$


Here, $F_{\perp}^{-\alpha}$ is the field strength tensor of the gluon. After integrating out $\vec{k}_{T}$, one obtains

$$
\widetilde{\Delta}_{A}^{\alpha}\left(z, \cos \theta, M_{h}^{2}, \phi_{R}\right)=\frac{z^{2}|\vec{R}|}{8 M_{h}} \int d^{2} \vec{k}_{T} \widetilde{\Delta}_{A}^{\alpha}\left(z, k_{T}, R\right) .
$$

The DiFF $\widetilde{G}^{\varangle}$ thus can be extracted from $\widetilde{\Delta}_{A}^{\alpha}\left(z, k_{T}, R\right)$ by the trace

$$
\frac{\varepsilon_{T}^{\alpha \beta} R_{T \beta}}{z} \widetilde{G}^{\varangle}\left(z, \cos \theta, M_{h}^{2}\right)=4 \pi \operatorname{Tr}\left[\widetilde{\Delta}_{A}^{\alpha}\left(z, \cos \theta, M_{h}^{2}, \phi_{R}\right) \gamma^{-} \gamma_{5}\right] .
$$

As shown in Ref. [17], we can expand the twist-3 DiFF $\widetilde{G}^{\varangle}$ up to the $p$-wave level as

$$
\widetilde{G}^{\varangle}\left(z, \cos \theta, M_{h}^{2}\right)=\widetilde{G}_{o t}^{\varangle}\left(z, M_{h}^{2}\right)+\widetilde{G}_{l t}^{\varangle}\left(z, M_{h}^{2}\right) \cos \theta .
$$

Here, $\widetilde{G}_{o t}^{\varangle}$ originates from the interference of $s$ and $p$ waves, and $\widetilde{G}_{l t}^{\varangle}$ comes from the interference of two $p$ waves with different polarization. The $\sin \phi_{R}$ asymmetry of dihadron production in the single longitudinally polarized SIDIS may be expressed as [14],

$$
A_{U L}^{\sin \phi_{R}}\left(x, z, M_{h}^{2}\right)=-\frac{\sum_{a} e_{a}^{2} \frac{|\vec{R}|}{Q}\left[\frac{|M|}{M_{h}} x h_{L}^{a}(x) H_{1, o t}^{\varangle, a}\left(z, M_{h}^{2}\right)+\frac{1}{z} g_{1}(x) \widetilde{G}_{o t}^{\varangle}\left(z, M_{h}^{2}\right)\right]}{\sum_{a} e_{a}^{2} f_{1}^{a}(x) D_{1, o o}^{a}\left(z, M_{h}^{2}\right)} .
$$

Following the COMPASS convention, the depolarization factors are not included in the numerator and denominator.

\section{Model calculation of $\widetilde{G}_{o t}^{\varangle}$}

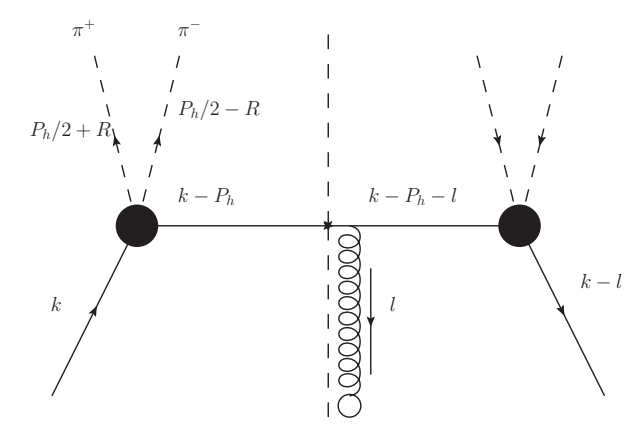

Figure 2: Diagrammatic representation of the correlation function $\widetilde{\Delta}_{A}^{\alpha}$ in the spectator model.

In the following, we present the calculation of unknown DiFF $\widetilde{G}_{\text {ot }}^{\varangle}$ in the same spectator model. The corresponding diagram for the calculation in the spectator model is shown in Fig. 2. The left hand side of Fig. 2 corresponds to the quark-hadron vertex $\left\langle P_{h} ; X|\bar{\psi}(0)| 0\right\rangle$, while the right hand side corresponds to the vertex containing gluon rescattering $\left\langle 0\left|i g F_{\perp}^{-\alpha}\left(\eta^{+}\right) \psi\left(\xi^{+}\right)\right| P_{h} ; X\right\rangle$. Therefore, the $s$ and $p$ wave contributions to the quark-gluon-quark correlator for dihardon fragmentation in the spectator model can be written as

$$
\widetilde{\Delta}_{A}^{\alpha}\left(k, P_{h}, R\right)=i \frac{C_{F} \alpha_{s}}{2(2 \pi)^{2}(1-z) P_{h}^{-}} \frac{1}{k^{2}-m^{2}} \int \frac{d^{4} l}{(2 \pi)^{4}}\left(l^{-} g_{T}^{\alpha \mu}-l_{T}^{\alpha} g^{-\mu}\right)
$$




$$
\frac{(\not \psi-\not l+m)\left(F^{s \star} e^{-\frac{k^{2}}{\Lambda_{s}^{2}}}+F^{p \star} e^{-\frac{k^{2}}{\Lambda_{p}^{2}}} R /\right)\left(\not /-P / h-\not l+m_{s}\right) \gamma_{\mu}\left(\not \not-P / h+m_{s}\right)\left(F^{s} e^{-\frac{k^{2}}{\Lambda_{s}^{2}}}+F^{p} e^{-\frac{k^{2}}{\Lambda_{p}^{2}}} R /\right)(\not \psi+m)}{\left(-l^{-} \pm i \varepsilon\right)\left((k-l)^{2}-m^{2}-i \varepsilon\right)\left(\left(k-P_{h}-l\right)^{2}-m_{s}^{2}-i \varepsilon\right)\left(l^{2}-i \varepsilon\right)},
$$

where $m$ and $m_{s}$ are the masses of the quark and the spectator, and where the factor $\left(l^{-} g_{T}^{\alpha \mu}-l_{T}^{\alpha} g^{-\mu}\right)$ comes from the Feynman rule corresponding to the gluon field strength tensor, as denoted by the open circle in Fig. 2. where $\Lambda_{s}$ and $\Lambda_{p}$ are the $z$-dependent $\Lambda$-cutoffs having the form [17]

$$
\Lambda_{s, p}=\alpha_{s, p} z^{\beta_{s, p}}(1-z)^{\gamma_{s, p}}
$$

and $2 / \Lambda_{s p}^{2}=1 / \Lambda_{s}^{2}+1 / \Lambda_{p}^{2}$. The on-shell condition of the spectator gives the relation between $k^{2}$ and the trasnverse momentum $\vec{k}_{T}$

$$
k^{2}=\frac{z}{1-z}\left|\vec{k}_{T}\right|^{2}+\frac{M_{s}^{2}}{1-z}+\frac{M_{h}^{2}}{z} .
$$

Thus, the final result for $\widetilde{G}_{o t}^{\varangle}\left(z, M_{h}^{2}\right)$ has the form

$$
\begin{aligned}
\widetilde{G}_{o t}^{\varangle}\left(z, M_{h}^{2}\right) & =\frac{\alpha_{s} C_{F} z^{2}|\vec{R}|}{8(2 \pi)^{4}(1-z) M_{h}} \frac{1}{k^{2}-m^{2}} \int d\left|\vec{k}_{T}\right|^{2} e^{-\frac{2 k^{2}}{\Lambda_{s p}^{2}}}\left\{\operatorname{Im}\left(F^{s *} F^{p}\right) C\right. \\
& \left.+\operatorname{Re}\left(F^{s *} F^{p}\right)\left(k^{2}-m^{2}\right) m_{s}\left[(A+z B)-I_{2}\right]\right\} .
\end{aligned}
$$

Here, the coefficients $C$ give

$$
C=\int_{0}^{1} d x \int_{0}^{1-x} d y \frac{-2 m\left[(x+y) k \cdot p-y M_{h}^{2}\right]+m\left(k^{2}-m^{2}\right)}{x(1-x) k^{2}+2 k \cdot\left(k-P_{h}\right) x y+m^{2} x+m_{s}^{2} y+y(y-1)\left(k-P_{h}\right)^{2}},
$$

where we can see that once $m=0$, so the $\operatorname{Im}\left(F^{s^{*}} F^{p}\right) C$ term will disappears, it has no effect on the results. and the coefficients $A$ and $B$ come from the decomposition of the integral [15],

$$
\int d^{4} l \frac{l^{\mu} \delta\left(l^{2}\right) \delta\left((k-l)^{2}-m^{2}\right)}{\left(k-P_{h}-l\right)^{2}-m_{s}^{2}}=A k^{\mu}+B P_{h}^{\mu}
$$

and have the expressions

$$
\begin{aligned}
& A=\frac{I_{1}}{\lambda\left(M_{h}, m_{s}\right)}\left(2 k^{2}\left(k^{2}-m_{s}^{2}-M_{h}^{2}\right) \frac{I_{2}}{\pi}+\left(k^{2}+M_{h}^{2}-m_{s}^{2}\right)\right), \\
& B=-\frac{2 k^{2}}{\lambda\left(M_{h}, m_{s}\right)} I_{1}\left(1+\frac{k^{2}+m_{s}^{2}-M_{h}^{2}}{\pi} I_{2}\right) .
\end{aligned}
$$

The functions $I_{i}$ appearing in the above equations are defined as [16].

\section{Numerical estimate}

In the following, we numerically estimate the $\sin \phi_{R}$ azimuthal asymmetry in the dihadron production off a longitudinally polarized proton by considering both the $h_{L} H_{1, o t}^{\varangle, a}$ term and the $g_{1} \widetilde{G}_{o t}^{\varangle}$ 
term. Using Eq. (2.6), we can obtain the expressions of the $x$-dependent, $z$-dependent and $M_{h^{-}}$ dependent $\sin \phi_{R}$ asymmetry as follows

$$
\begin{gathered}
A_{U L}^{\sin \phi_{R}}(x)=-\frac{\int d z \int d M_{h} 2 M_{h} \frac{|\vec{R}|}{Q}\left[\frac{|M|}{M_{h}}\left(4 h_{L}^{u}(x)+h_{L}^{d}(x)\right) x H_{1, o t}^{\varangle}\left(z, M_{h}^{2}\right)+\frac{1}{z}\left(4 g_{1}^{u}(x)+g_{1}^{d}(x)\right) \widetilde{G}_{o t}^{\varangle}\left(z, M_{h}^{2}\right)\right]}{\int d z \int d M_{h} 2 M_{h}\left(4 f_{1}^{u}(x)+f_{1}^{d}(x)\right) D_{1, o o}\left(z, M_{h}^{2}\right)}, \\
A_{U L}^{\sin \phi_{R}}(z)=-\frac{\int d x \int d M_{h} 2 M_{h} \frac{|\vec{R}|}{Q}\left[\frac{M \mid}{M_{h}}\left(4 h_{L}^{u}(x)+h_{L}^{d}(x)\right) x H_{1, o t}^{\varangle}\left(z, M_{h}^{2}\right)+\frac{1}{z}\left(4 g_{1}^{u}(x)+g_{1}^{d}(x)\right) \widetilde{G}_{o t}^{\varangle}\left(z, M_{h}^{2}\right)\right]}{\int d x \int d M_{h} 2 M_{h}\left(4 f_{1}^{u}(x)+f_{1}^{d}(x)\right) D_{1, o o}\left(z, M_{h}^{2}\right)}, \\
A_{U L}^{\sin \phi_{R}}\left(M_{h}\right)=-\frac{\int d x \int d z \frac{|\vec{R}|}{Q}\left[\frac{M \mid}{M_{h}}\left(4 h_{L}^{u}(x)+h_{L}^{d}(x)\right) x H_{1, o t}^{\varangle}\left(z, M_{h}^{2}\right)+\frac{1}{z}\left(4 g_{1}^{u}(x)+g_{1}^{d}(x)\right) \widetilde{G}_{o t}^{\varangle}\left(z, M_{h}^{2}\right)\right]}{\int d x \int d z\left(4 f_{1}^{u}(x)+f_{1}^{d}(x)\right) D_{1, o o}\left(z, M_{h}^{2}\right)} .
\end{gathered}
$$

For the other DiFFs $H_{1, o t}^{\varangle, a}\left(z, M_{h}^{2}\right)$ and $D_{1, o o}\left(z, M_{h}^{2}\right)$ needed in the calculation, we apply the same spectator model results from Ref. [17]. For the twist-3 distribution $h_{L}$, we choose the result in Ref. [18], as for the twist-2 PDFs $f_{1}$ and $g_{1}$, we adopt the results calculated from the same model [19] for consistency. To compare estimate the $\sin \phi_{R}$ asymmetry in SIDIS at COMPASS, we adopt the following kinematical cuts [14]

$$
\begin{aligned}
& 0.003<x<0.4, \quad 0.1<y<0.9, \quad 0.2<z<0.9 \\
& 0.3 \mathrm{GeV}<M_{h}<1.6 \mathrm{GeV}, \quad Q^{2}>1 \mathrm{GeV}^{2}, \quad W>5 \mathrm{GeV} .
\end{aligned}
$$

In Fig. 3, we plot the $\sin \phi_{R}$ asymmetry in dihadron production off the longitudinally polarized
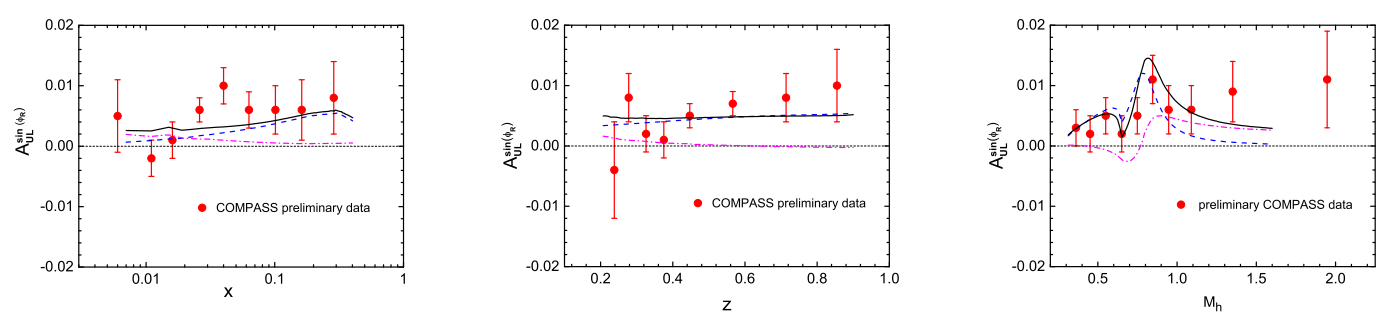

Figure 3: The $\sin \phi_{R}$ azimuthal asymmetry in dihadron production off the longitudinally polarized proton as functions of $x$ (left panel), $z$ (central panel) and $M_{h}$ (right panel) at COMPASS. The full circles show the COMPASS preliminary data [14] for comparison. The dashed curves denote the contribution from the $h_{L} H_{1, o t}^{\varangle}$ term, the dashed-dotted curves represent the contribution from the $g_{1} \widetilde{G}^{\varangle}$ term, and the solid lines display the sum of two contributions.

proton at the kinematics of COMPASS. The $x$-, $z$ - and $M_{h}$-dependent asymmetries are depicted in the left panel, central, and right panels of the figure. We find that in the large $x$ region and in the small $M_{h}$ region, the contribution from the $h_{L} H_{1, o t}^{\varangle, a}$ term dominates the asymmetry. The $g_{1} \widetilde{G}^{\varangle}$ becomes important in the small $x$ region and large $M_{h}$ region. Combining the contributions from the two terms, our calculation agrees with the COMPASS preliminary data on the $\sin \phi_{R}$ asymmetry. 


\section{Conclusion}

In this work, we have studied the single longitudinal-spin asymmetry with a $\sin \phi_{R}$ modulation of dihadron production in SIDIS. We found that the contribution to $\widetilde{G}_{o t}^{\varangle}$ comes from the interference of the $s$ and $p$ waves. Using the numerical results of the DiFFs, we estimated the $\sin \phi_{R}$ asymmetry and compared it with the COMPASS measurement. Our calculation shows that the $h_{L} H_{1, \text { ot }}^{\varangle}$ term dominates in the most of the kinematical region. However, the inclusion of the $g_{1} G_{o t}^{\varangle}$ contribution yields a better description of the COMPASS data, especially in the large $M_{h}$ region.

\section{References}

[1] A. Kotzinian, Nucl. Phys. B441, 234 (1995).

[2] A. Bacchetta, M. Diehl, K. Goeke, A. Metz, P. J. Mulders and M. Schlegel, J. High Energy Phys. 0702,093 (2007).

[3] K. Konishi, A. Ukawa, and G. Veneziano, Phys. Lett. B 78, 243 (1978).

[4] I. Vendramin, Nuovo Cim. A 66, 339 (1981).

[5] U. P. Sukhatme and K. E. Lassila, Phys. Rev. D 22, 1184 (1980).

[6] A. Majumder and X. N. Wang, Phys. Rev. D 70, 014007 (2004), [hep-ph/0402245].

[7] A. Bianconi, S. Boffi, R. Jakob and M. Radici, Phys. Rev. D 62, 034008 (2000) [hep-ph/9907475].

[8] M. Radici, R. Jakob and A. Bianconi, Phys. Rev. D 65, 074031 (2002) [hep-ph/0110252].

[9] A. Bacchetta and M. Radici, Phys. Rev. D 67, 094002 (2003) [hep-ph/0212300].

[10] M. Radici, A. M. Ricci, A. Bacchetta and A. Mukherjee, Phys. Rev. D 94, 034012 (2016) [arXiv:1604.06585 [hep-ph]].

[11] M. Radici and A. Bacchetta, Phys. Rev. Lett 120, 192001 (2018)

[12] A. Courtoy, A. Bacchetta, M. Radici and A. Bianconi, Phys. Rev. D 85, 114023 (2012) [arXiv:1202.0323 [hep-ph]].

[13] A. Bacchetta and M. Radici, Phys. Rev. D 69, 074026 (2004), [hep-ph/0311173].

[14] S. Sirtl, in 22nd International Symposium on Spin Physics (SPIN 2016) Urbana, IL, USA (unpublished) arXiv: 1702.07317, http://www.indiana.edu/spin2016/.

[15] Z. Lu and I. Schmidt, Phys. Lett. B 747, 357 (2015), [arXiv:1501.04379 [hep-ph]].

[16] D. Amrath, A. Bacchetta and A. Metz, Phys. Rev. D 71, 114018 (2005), arXiv:hep-ph/0504124.

[17] A. Bacchetta and M. Radici, Phys. Rev. D 74, 114007 (2006), [hep-ph/0608037].

[18] Z. Lu, Phys. Rev. D 90, 014037 (2014), [hep-ph/1404.4229].

[19] A. Bacchetta, F. Conti and M. Radici, Phys. Rev. D 78, 074010 (2008) [arXiv:0807.0323 [hep-ph]]. 